\title{
A Comparison of Alignment Models for Statistical Machine Translation
}

\author{
Franz Josef Och and Hermann Ney \\ Lehrstuhl für Informatik VI, Computer Science Department \\ RWTH Aachen - University of Technology \\ D-52056 Aachen, Germany \\ \{och, ney\}@informatik.rwth-aachen.de
}

\begin{abstract}
In this paper, we present and compare various alignment models for statistical machine translation. We propose to measure the quality of an alignment model using the quality of the Vitcrbi alignment compared to a manually-produced alignment and describe a refined annotation scheme to produce suitable reference alignments. We also compare the impact of different alignment models on the translation quality of a statistical machine translation system.
\end{abstract}

\section{Introduction}

In statistical machine translation (SMT) it is necessary to model the translation probability $\operatorname{Pr}\left(f_{1}^{J} \mid e_{1}^{I}\right)$. Here $f_{1}^{I}=\mathbf{f}$ denotes the (French) source and $e_{1}^{I}=\mathbf{e}$ denotes the (English) target string. Most SMT models (Brown et al., 1993; Vogel ct al., 1996) try to model word-to-word correspondences between sourcc and target words using an alignment mapping from source position $j$ to target position $i=a_{j}$.

We can rewrite the probability $\operatorname{Pr}\left(f_{1}^{I} \mid e_{1}^{I}\right)$ by introducing the 'hidden' alignments $a_{1}^{J}:=a_{1} \ldots a_{j} \ldots a_{J}$ $\left(a_{j} \in\{0, \ldots, I\}\right)$ :

$$
\begin{aligned}
\operatorname{Pr}\left(f_{1}^{J} \mid e_{1}^{I}\right) & =\sum_{a_{1}^{J}} \operatorname{Pr}\left(f_{1}^{J}, a_{1}^{J} \mid e_{1}^{I}\right) \\
& =\sum_{a_{1}^{J}} \prod_{j=1}^{J} \operatorname{Pr}\left(f_{j}, a_{j} \mid f_{1}^{j-1}, a_{1}^{j-1}, e_{1}^{I}\right)
\end{aligned}
$$

To allow for French words which do not dircctly correspond to any English word an artificial 'empty' word $e_{0}$ is added to the target sentence at position $i=0$.

The different alignment models we present provide different decompositions of $\operatorname{Pr}\left(f_{1}^{J}, a_{1}^{J} \mid e_{1}^{I}\right)$. An alignment $\hat{a}_{1}^{J}$ for which holds

$$
\hat{a}_{1}^{J}=\arg \max _{a_{1}^{J}} \operatorname{Pr}\left(f_{1}^{J}, a_{1}^{J} \mid e_{1}^{I}\right)
$$

for a specific model is called Viterbi alignment of this model.

In this paper we will describe extensions to the Hidden-Markov alignment model from (Vogel et al.,
1996) and compare these to Models 1 - 4 of (Brown et al., 1993). We propose to mcasurc the quality of an alignment model using the quality of the Viterbi alignment compared to a manually-produced alignment. This has the advantage that once having produced a reference alignment, the evaluation itsolf can be performed automatically. In addition, it results in a very precise and reliable cvaluation criterion which is well suited to assess various design decisions in modeling and training of statistical alignmont modcls.

It is well known that manually performing a word alignment is a complicated and ambiguous task (Mclamed, 1998). Therefore, to produce the roforcnce alignment wo use a refined annotation scheme which reduces the complications and ambiguities occurring in the manual construction of a word alignment. As we use the alignment models for machine translation purposes, we also cvaluate the resulting translation quality of different models.

\section{Alignment with HMM}

In the Hidden-Markov alignment model we assume a first-order dependence for the alignments $a_{j}$ and that the translation probability depends only on $a_{j}$ and not on $a_{j-1}$ :

$$
\operatorname{Pr}\left(f_{j}, a_{j} \mid f_{1}^{j-1}, a_{1}^{j-1}, c_{1}^{I}\right)=p\left(a_{j} \mid a_{j-1}, I\right) p\left(f_{j} \mid e_{a_{j}}\right)
$$

Later, we will describe a refinement with a depondence on $e_{a_{j-1}}$ in the alignment model. Putting everything together, we have the following basic HMM-based model:

$$
p\left(f_{1}^{J} \mid e_{1}^{I}\right)=\sum_{a_{1}^{J}} \prod_{j=1}^{J}\left[p\left(a_{j} \mid a_{j-1}, I\right) \cdot p\left(f_{j} \mid e_{a_{j}}\right)\right]
$$

with the alignment probability $p\left(i \mid i^{\prime}, I\right)$ and the translation probability $p(f \mid e)$. To find a Viterbi alignment for the HMM-based model we resort to dynamic programming (Vogel et al., 1996).

The training of the HMM is done by the EMalgorithm. In the E-step the lexical and alignment 
counts for one sentence-pair $(f, c)$ are calculated:

$$
\begin{gathered}
c(f \mid \mathbf{c} ; \mathbf{f}, \mathbf{e})=\sum_{\mathbf{a}} \operatorname{Pr}(\mathbf{a} \mid \mathbf{f}, \mathbf{c}) \sum_{i, j} \delta\left(f, f_{j}\right) \delta\left(c, \boldsymbol{c}_{i}\right) \\
c\left(i \mid i^{\prime}, I ; \mathbf{f}, \mathbf{c}\right)=\sum_{\mathbf{a}} \operatorname{Pr}(\mathbf{a} \mid \mathbf{f}, \mathbf{c}) \sum_{j} \delta\left(i^{\prime}, a_{j-1}\right) \delta\left(i, a_{j}\right)
\end{gathered}
$$

In the M-step the lexicon and translation probabilities are:

$$
\begin{aligned}
p(f \mid e) & \propto \sum_{s} c\left(f \mid e ; \mathbf{f}^{(s)}, \mathbf{e}^{(s)}\right) \\
p\left(i \mid i^{\prime}, I\right) & \propto \sum_{s} c\left(i \mid i^{\prime}, I ; \mathbf{f}^{(s)}, \mathbf{e}^{(s)}\right)
\end{aligned}
$$

To avoid the summation over all possible alignments a, (Vogel ot al., 1996) use the maximum approximation where only the Viterbi alignment path is used to collect counts. Wo used the Baum-Wolch-algorithm (Baum, 1972) to train the modol parameters in our cxperiments. Thereby it is possible to perform an ofliciont training using all alignmonts.

To make the alignment parameters independent from absolute word positions we assume that the alignment probabilities $p\left(i \mid i^{\prime}, I\right)$ depend only on the jump width $\left(i-i^{\prime}\right)$. Using a set of non-negative parameters $\left\{c\left(i-i^{\prime}\right)\right\}$, we can write the alignment, probabilities in the form:

$$
p\left(i \mid i^{\prime}, I\right)=\frac{c\left(i-i^{\prime}\right)}{\sum_{i^{\prime \prime}=-1}^{I} c\left(i^{\prime \prime}-i^{\prime}\right)} .
$$

This form ensures that for ade word position $i^{\prime}$, $i^{\prime}=1, \ldots, I$, the alignment, probabilities satisfy the normalization constraint.

\section{Extension: refined alignment model}

The count table $c\left(i-i^{\prime}\right)$ has only $2 \cdot I_{\text {man }}-1$ entries. This might be suitable for small corpora, but for large corpora it is possible to make a more refined model of $\operatorname{Pr}\left(a_{j} \mid f_{1}^{j-1}, a_{1}^{j-1}, c_{1}^{I}\right)$. Especially, we analyzed the effect of a dependence on $e_{a_{j-1}}$ or $f_{j}$. $A_{\text {is }}$ a dependence on all English words would jesult in a huge number of alignment paranneters we use as (Brown et al., 1993) equivalence classes $G$ over the English and the French words. Here $G$ is a mapping of words to classes. This mapping is trained antomatically using a modification of the method described in (Kneser and Ney, 1991). Wo use 50 classes in our experiments. The most general form of alignment distribution that, we consider in the IIMM is $p\left(a_{j}-a_{j-1} \mid G\left(c_{a_{j}}\right), G\left(f_{j}\right), I\right)$.

Extension: empty word

In the original formulation of the HMM alignment model there is no 'empty' word which gencrates Fronch words having no directly aligned English word. A direct inclusion of an empty word in the
HMM model by adding an $e_{0}$ as in (Brown et al., 1.993 ) is not possible if we want to model the jump distances $i-i^{\prime}$, as the position $i=0$ of the cmpty word is chosen arbitrarily. Therefore, to introduce the empty word we extend the HMM network by $I$ empty words $e_{I+1}^{2 I}$. Tho English word $e_{i}$ has a corresponding empty word $e_{i+l}$. The position of the empty word encodes the previously visited English word.

We enforce the following constraints for the transitions in the HMM network $\left(i \leq I, i^{I} \leq I\right)$ :

$$
\begin{aligned}
p\left(i+I \mid i^{\prime}, I\right) & =p_{0}^{I I} \cdot \delta\left(i, i^{\prime}\right) \\
p\left(i+I \mid i^{\prime}+I, I\right) & =p_{0}^{I I} \cdot \delta\left(i, i^{\prime}\right) \\
p\left(i \mid i^{\prime}+I, I\right) & =p\left(i \mid i^{\prime}, I\right)
\end{aligned}
$$

The parameter $p_{0}^{I I}$ is the probability of a transition to the empty word. In our experiments we set $\eta_{0}^{I I}=$ 0.2 .

\section{Smoothing}

For a better estimation of infrequent events we introduce the following smoothing of alignment probabilitios:

$$
p^{\prime}\left(a_{j} \mid a_{j-1}, I\right)=\alpha \cdot \frac{1}{I}+(1-\alpha) \cdot p\left(a_{j} \mid a_{j-1}, I\right)
$$

In our experiments we use $\alpha=0.4$.

\section{$3 \quad$ Model 1 and Model 2}

Replacing the dependence on $a_{j-1}$ in the HMM alignment model by a dependence on $j$, we obtain a model which can be seen as a zero-order HiddenMarkov Morlel which is similar to Moctel 2 proposed by (Brown ot al., 1993). Assuming a uniform alignment probability $p(i \mid j, I)=1 / 1$, we obtain Model 1.

Assuming that the dominating factor in the alignment model of Model 2 is the distance relative to the diagonal line of the $(j, i)$ plane the model $p(i \mid j, I)$ can be structured as follows (Vogel ot al., 1996):

$$
p(i \mid j, I)=\frac{r\left(i-j \frac{I}{J}\right)}{\sum_{i^{\prime}=1}^{I} r\left(i^{\prime}-j \frac{I}{J}\right)} .
$$

This model will be referred to as diagonal-oriented Model 2.

\section{Model 3 and Model 4}

Model: The fertility models of (Brown et al., 1993) explicitly model the probability $p(\phi \mid e)$ that the English word $e_{i}$ is aligned to

$$
\phi_{i}=\sum_{j} \delta\left(a_{j}, i\right)
$$

French words. 
Model 3 of (Brown et al., 1993) is a zero-order alignmont model like Model 2 including in addition fertility parameters. Model 4 of (Brown ct al., 1993 ) is also a first-order alignment model (along the source positions) like the HMM, but includes also fertilities. In Model 4 the alignment position $j$ of an English word depends on the alignment position of the previous English word (with non-zero fertility) $j^{\prime}$. It models a jump distance $j-j^{\prime}$ (for consecutive English words) while in the HMM a jump distance $i-i^{\prime}$ (for consecutive French words) is modeled. The full description of Model 4 (Brown et al., 1993 ) is rather complicated as there have to be considered the cases that English words have fertility larger than one and that English words have fortility zero.

For training of Model 3 and Model 4, we use an cxtension of the program GrzA (Al-Onaizan et al., 1999). Since there is no efficient way in these models to avoid the explicit summation over all alignments in the EM-algorithm, the counts are collected only over a subset of promising alignments. It is not known an efficient algorithm to compute the Viterbi alignment for the Models 3 and 4. Therefore, the Viterbi alignment is computed only approximately using the method described in (Brown et al., 1993). The models 1-4 are trained in succession with the final parameter values of one model serving as the starting point for the next.

A special problem in Model 3 and Model 4 concerns the deficiency of the model. This results in problems in re-estimation of the paraneter which describes the fertility of the empty word. In normal EM-training, this parameter is steadily decreasing, producing too many alignmonts with the empty word. Therefore we set the probability for aligning a source word with the empty word at a suitably chosen constant value.

As in the HMM wo easily can extend the dopendencies in the alignment model of Model 4 easily using the word class of the previous English word $E=G\left(e_{i^{\prime}}\right)$, or the word class of the French word $F=G\left(f_{j}\right)$ (Brown et al., 1993).

\section{Including a Manual Dictionary}

We propose here a simple method to make use of a bilingual dictionary as an additional knowledge source in the training process by extending the training corpus with the dictionary entries. Thoroby, the dictionary is used already in EM-training and can improve not only the alignment for words which are in the dictionary but indirectly also for other words. The additional sentences in the training corpus are weighted with a factor $F_{l e x}$ during the EM-training of the lexicon probabilities.

We assign the dictionary entries which really cooccur in the training corpus a high weight $F_{l e x}$ and the remaining entries a very low weight. In our experiments we use $F_{l e x}=10$ for the co-occurring dictionary entries which is equivalent to adding every dictionary entry ten times to the training corpus.

\section{The Alignment Template System}

The statistical machinc-translation method described in (Och et al., 1999) is based on a word aligned training corpus and thereby makes use of singleword based alignment models. The key element of this approach are the alignment templates which are pairs of phrases together with an alignment between the words within the phrases. The advantage of the alignment template approach over word based statistical translation models is that word context and local re-orderings are explicitly taken into account. We typically observe that this approach produces better translations than the single-word based models. The alignment templates are automatically trained using a parallel training corpus. For more information about the alignment template approach sec (Och et al., 1999).

\section{Results}

We present results on the Verbmobil Task which is a speech translation task in the domain of appointment scheduling, travel planning, and hotel reservation (Wahlster, 1993).

We measure the quality of the above mentioned alignment models with respect to alignment quality and translation quality.

To obtain a reforence alignment for cvaluating alignment quality, we manually aligned about 1.4 percent of our training corpus. We allowed the humans who performed the alignment to specify two different kinds of alignments: an S (sure) alignment which is used for alignments which are unambiguously and a $\mathrm{P}$ (possible) alignment which is used for alignments which might or might not exist. The $P$ relation is used especially to align words within idiomatic expressions, free translations, and missing function words. It is guaranteed that $S \subseteq P$. Figure 1 shows an example of a manually aligned sentence with S and P relations. The human-annotated alignment does not prefer any translation direction and may therefore contain many-to-one and one-to-many relationships. The annotation has been performed by two annotators, producing sets $S_{1}, P_{1}, S_{2}, P_{2}$. The reference alignment is produced by forming the intersection of the sure alignments $\left(S=S_{1} \cap S_{2}\right)$ and the union of the possible alignments $\left(P=P_{1} \cup P_{2}\right)$.

The quality of an aligmment $A=\left\{\left(j, a_{j}\right)\right\}$ is measured using the following alignment error rate:

$$
A E R(S, P ; A)=1-\frac{|A \cap S|+|A \cap P|}{|A|+|S|} .
$$




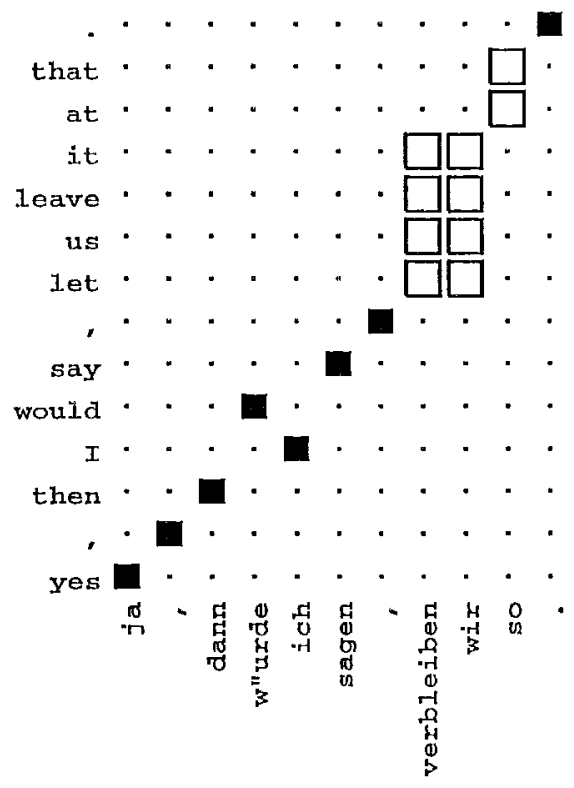

Figure 1: Ixample of a mamually amotated alignment with sure (filled dots) and possible connections.

Obviously, if we compare the sure alignments of evcry single annotator with the reference alignment we obtain an AER of zero percent.

Table 1: Corpus characteristics for alignment quality cxperiments.

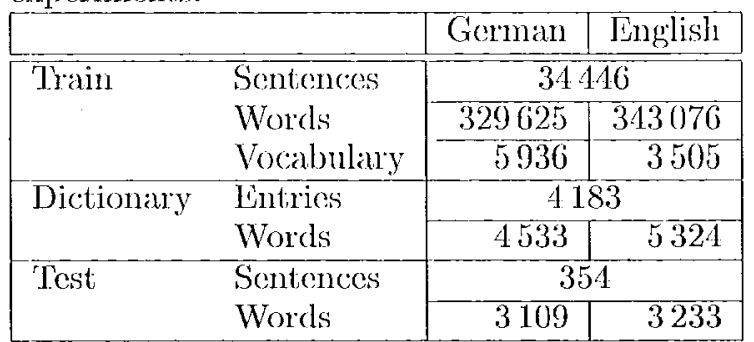

Table 1 shows the characteristics of training and test corpus used in the alignment quality experiments. The test corpus for these experiments (not for the translation experiments) is part of the training corpus.

Table 2 shows the aligmment quality of different alignment models. Here the alignment models of FMM and Model 4 do not include a dependence on word classes. We conclude that more sophisticated alignment models are crucial for good alignment quality. Consistently, the use of a first-order alignment model, modeling an empty word and fertilities result in better alignments. Interestingly, the simpler HMM alignment model outperforms Model 3 which shows the importance of first-order alignment models. The best, performance is achieved with Model 4 . 'The improvenent by using a dictio- nary is small compared to the effect of using better alignment models. We see a significant difforence in alignment quality if we exchange source and target languages. This is due to the restriction in all alignment models that a source language word can be aligned to at, most one target language word. If German is source language the frequently occurring German word compounds, cannot be aligned correctly, as they typically correspond to two or more English words.

Table 3 shows the effect of including a dependence on word classes in the alignment model of HMM or Model 4. By using word classes the results can be

Table 3: Effect of including a dependence on word classes in the alignment model.

\begin{tabular}{|l|c|c|}
\hline & \multicolumn{2}{|c|}{ AER [\%] } \\
\hline Dependencies & HMM & Model 4 \\
\hline no & 8.0 & 6.5 \\
source & 7.5 & 6.0 \\
target & 7.1 & 6.1 \\
source + target; & 7.6 & 6.1 \\
\hline
\end{tabular}

improved by $0.9 \%$ when using the IHMM and by $0.5 \%$ when using Model 4.

For the translation experiments wo used a difforont training and an independent tost corpus (Table 4).

Table 4: Corpus characteristics for translation quality experiments.

\begin{tabular}{|cl|r|r|}
\hline \multirow{2}{*}{ Train } & German & English \\
\cline { 2 - 3 } & Sentences & \multicolumn{2}{|c|}{58332} \\
\cline { 2 - 3 } Test & Vocds & 510523 & 549921 \\
\cline { 2 - 3 } & Sentences & 7940 & 4673 \\
& Words & \multicolumn{2}{|c|}{147} \\
\cline { 2 - 3 } & PP (trigram LM) & 1968 & 2173 \\
\hline
\end{tabular}

For the evaluation of the translation quality we used the automatically computable Word Error Rate (WER) and the Subjective Sentence Error Rate (SSER) (Nießen et al., 2000). The WER corresponds to the edit distance between the produced translation and one predefined reference translation. To obtain the SSER the translations are classified by human experts into a small number of quality classes ranging from "perfect" to "absolutely wrong". In comparison to the WER, this criterion is more meaningful, but it is also very expensive to measure. The translations are produced by the alignment template system mentioned in the previous section. 
Table 2: Alignment error rate (AER [\%]) of different alignment models for the translations directions English into German (German words have fertilities) and German into English.

\begin{tabular}{|l|c|c|c||c|c|c|}
\hline & \multicolumn{3}{|c|}{ English $\rightarrow$ Gorman } & \multicolumn{3}{|c|}{ German $\rightarrow$ English } \\
\hline Dictionary & \multicolumn{2}{|c|}{ no } & yes & \multicolumn{2}{|c|}{ no } & yes \\
\hline Empty Word & no & yes & yes & no & yes & yes \\
\hline \hline Model 1 & 17.8 & 16.9 & 16.0 & 22.9 & 21.7 & 20.3 \\
Model 2 & 12.8 & 12.5 & 11.7 & 17.5 & 17.1 & 15.7 \\
Model 2(diag) & 11.8 & 10.5 & 9.8 & 16.4 & 15.1 & 13.3 \\
Model 3 & 10.5 & 9.3 & 8.5 & 15.7 & 14.5 & 12.1 \\
HMM & 10.5 & 9.2 & 8.0 & 14.1 & 12.9 & 11.5 \\
Model 4 & 9.0 & 7.8 & 6.5 & 14.0 & 12.5 & 10.8 \\
\hline
\end{tabular}

Table 5: Effect of different alignment models on translation quality.

\begin{tabular}{|l|c|c|}
\hline $\begin{array}{l}\text { Alignment Model } \\
\text { in Training }\end{array}$ & WER[\%] & SSER[\%] \\
\hline \hline Model 1 & 49.8 & 22.2 \\
\hline HMM & 47.7 & 19.3 \\
\hline Model 4 & 48.6 & 16.8 \\
\hline
\end{tabular}

The results are shown in Table 5 . We sce a clear improvement in translation quality as measured by SSER whereas WER is more or less the same for all models. The improvement is due to better loxicons and better alignment tomplates extracted from the resulting alignments.

\section{Conclusion}

We have evaluated various statistical alignment models by comparing the Viterbi alignment of the model with a human-made alignment. We have shown that by using more sophisticated models the quality of the alignments improves significantly. Further improvements in producing better alignments are expected from using the HMM alignment model to bootstrap the fertility models, from making use of cognates, and from statistical alignment modols that are based on word groups rather than single words.

\section{Acknowledgment}

This article has been partially supported as part of the Verbmobil project (contract number 01 IV 701 T4) by the German Federal Ministry of Education, Science, Research and Technology.

\section{References}

Y. Al-Onaizan, J. Curin, M. Jahr, K. Knight, J. Lafferty, I. D. Mclamed, F. J. Och, D. Purdy, N. A.
Smith, and D. Yarowsky. 1999. Statistical machinc translation, final report, JHU workshop). http://www.clsp.jhu.edu/ws99/projects/mt/ final report/mt-final-report.ps.

L.E. Baum. 1972. An Inequality and Associated Maximization Technique in Statistical Estimation for Probabilistic Functions of Markov Processes. Inequalities, 3:1-8.

P. F. Brown, S. A. Della Pietra, V. J. Della Pictra, and R. L. Mercer. 1993. The mathematics of statistical machine translation: Parameter estimation. Computational Linguistics, 19(2):263-311.

R. Kneser and H. Noy. 1991. Forming Word Classes by Statistical Clustering for Statistical Language Modelling. In 1. Quantitative Linguistics Conf.

I. D. Melamed. 1998. Manual annotation of translational equivalence: 'The Blinker project. Technical Report 98-07, IRCS.

S. Nieben, F. J. Och, G. Leusch, and H. Ney. 2000. An cvaluation tool for machine translation: Fast cvaluation for mt research. In Proceedings of the Second International Conference on Language Resources and Evaluation, pages 39-45, Athens, Greece, May Junc.

F. J. Och, C. Tillmann, and H. Ney. 1999. Improved alignment models for statistical machine translation. In In Proc. of the Joint SIGDAT Conf. on Empirical Methods in Natural Language Processing and Very Larye Corpora, pages 20-28, University of Maryland, College Park, MD, USA, June.

S. Vogel, H. Ney, and C. Tillmann. 1996. HMMbased word alignment in statistical translation. In COLING '96: The 16th Int. Conf. on Computational Linguistics, pages 836-841, Copenhagen, August.

W. Wahlster. 1993. Verbmobil: Translation of faceto-face dialogs. In Proc. of the MT Summit IV, pages 127-135, Kobe, Japan. 